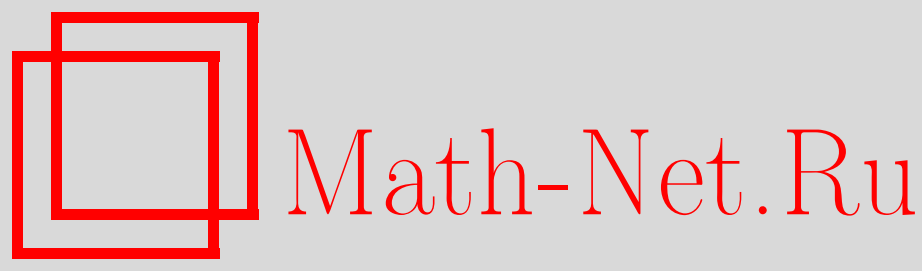

Е. Е. Тареева, Т. И. Щелкачёва, Н. М. Щелкачёв, Ориентационное упорядочение и переход в состояние ориентационного стекла в фуллерите $\mathrm{C}_{60}, T M \Phi, 2008$, том 155 , номер 2, 356-368

DOI: https://doi.org/10.4213/tmf6217

Использование Общероссийского математического портала Math-Net.Ru подразумевает, что вы прочитали и согласны с пользовательским соглашением http://www . mathnet.ru/rus/agreement

Параметры загрузки:

IP: 54.166 .219 .16

26 апреля 2023 г., 15:51:14

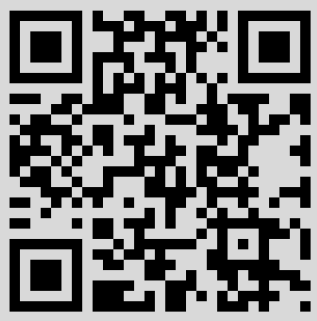




\title{
ФИЗИКА
}

Том 155, № 2

май, 2008

2008 г. Е. Е. Тареева*, Т. И. Щелкачева*, Н. М. Щелкачев*

\section{ОРИЕНТАЦИОННОЕ УПОРЯДОЧЕНИЕ И ПЕРЕХОД В СОСТОЯНИЕ ОРИЕНТАЦИОННОГО СТЕКЛА В ФУЛЛЕРИТЕ $\mathrm{C}_{60}$}

\begin{abstract}
Предложена модель для описания низкотемпературного перехода в состояние ориентационного стекла в твердом молекулярном $\mathrm{C}_{60}$ в духе теории спиновых стекол. Найдено репликосимметричное решение, а также проведено нарушение репличной симметрии. Полученные результаты находятся в согласии с экспериментальными данными о частичном сохранении ориентационного дальнего порядка в фазе стекла и о наличии широкого максимума на кривой зависимости ориентационной части теплоемкости от температуры.
\end{abstract}

Ключевые слова: фуллерен, ориентационный дальний порядок, ориентационное стекло, спиновые стекла.

\section{1. ВВЕДЕНИЕ}

Как известно, молекулы $\mathrm{C}_{60}$ имеют близкую к сферической форму и связаны между собой достаточно слабыми силами типа сил Ван-дер-Ваальса. Тем не менее ориентационные степени свободы играют определяющую роль в ряде структурных и физических свойств фуллерита (см., например, обзоры [1], [2]). При понижении температуры до величины ниже комнатной в нем происходят два ориентационных фазовых перехода - переход в состояние с ориентационным дальним порядком (типа антиферромагнитного состояния) при $260 \mathrm{~K}$ и переход в состояние ориентационного стекла при $90 \mathrm{~K}$. Кроме того, на кривой теплоемкости при $150 \mathrm{~K}$ имеется некоторая особенность, которую также связывают обычно с ориентационными степенями свободы. В настоящей работе мы будем заниматься микроскопической теорией ориентационного стекла.

В молекуле фуллерена 60 эквивалентных атомов углерода расположены в вершинах усеченного икосаэдра, состоящего из 12 пентагонов и 20 гексагонов. Каждый атом связан с тремя другими углеродными атомами посредством двух одинарных и одной двойной связи: шестиугольники образованы по типу бензольного кольца с двойными связями и избытком заряда на них. Недостаток заряда при этом возникает в центрах пятиугольников и шестиугольников (см., например, [3]-[5]). При

* Институт физики высоких давлений, г. Троицк, Московская обл., Россия. E-mail: nms@itp.ac.ru 
комнатной и более высоких температурах молекулы фуллерена можно рассматривать как однородные сферы. Фуллерен кристаллизуется в гранецентрированную кубичесую (ГЦК) решетку с четырьмя эквивалентными молекулами в ячейке.

Для изучения возможных ориентационных упорядочений весьма существенно, что молекула $\mathrm{C}_{60}$ устроена так, что можно одновременно направить 6 из ее пентагонов или 6 из ее гексагонов на двойные связи в шести ее ближайших соседях в ГКЦ-решетке (соответственно Р- или Н-состояния), а на оставшиеся 6 ближайших соседей направить 6 из ее межпентагонных двойных связей. Эти Р- и Н-состояния являются наиболее выгодными взаимными ориентациями молекул, и, как показывает эксперимент, при понижении температуры до величин порядка комнатной свободное вращение молекул сменяется быстрыми прыжками между этими состояниями. На этом факте и основана наша дискретизированная модель.

При понижении температуры в результате фазового перехода первого рода при $T_{\mathrm{c}} \approx 260 \mathrm{~K}$ возникает дальний ориентационный порядок. Исходная ГКЦ-решетка разделяется на 4 простые кубические подрешетки, в каждой из которых имеет место своя преимущественная ориентация молекул. Происходит понижение симметрии до $P a \overline{3}$ [6]-[8]. При этом остается динамический беспорядок. Каждая молекула переориентируется за счет быстрых прыжков между двумя наиболее выгодными для нее Р- и Н-положениями. Доля наиболее выгодной Р-ориентации при нормальном давлении меняется от $60 \%$ при $T=260 \mathrm{~K}$ до $84 \%$ при $T_{\mathrm{g}}=90 \mathrm{~K}$, когда происходит переход в ориентационное стекло. Ниже этой температуры, в состоянии ориентационного стекла, изменение относительного количества Р- и Н-состояний прекращается [1], [2], [9]-[14]. Обычно считают, что замерзание ориентационных степеней свободы происходит тогда, когда температура становится слишком низкой для преодоления потенциального барьера между энергетически выгодными Р- и Н-положениями. При этом наблюдается плавный максимум на кривой температурной зависимости ориентационного вклада в теплоемкость [9], [15], [16] и имеет место характерное для переходов в стекло увеличение времен релаксации. Одним из наиболее интересных свойств этого перехода является тот факт, что ориентационное стекло сосуществует с частично сохранившимся дальним ориентационным порядком.

Обычно состояние ориентационного стекла в $\mathrm{C}_{60}$ описывается чисто феноменологически как "замерзание" ансамбля двухуровневых систем [9], [17]. Однако нам представляется естественным попробовать найти аналогию между состоянием стекла в $\mathrm{C}_{60}$ и обычными спиновыми стеклами, а точнее ориентационными стеклами в системах с более простыми, чем $\mathrm{C}_{60}$, молекулами такими, как смесь орто- и пара-водорода или смесь $\mathrm{N}_{2}-\mathrm{Ar}$. Цель нашей работы - попытаться описать низкотемпературное ориентационное стекло в $\mathrm{C}_{60}$ на микроскопическом уровне в духе теории спиновых стекол, основываясь на наших предыдущих работах: с одной стороны, на достаточно успешном описании квадрупольных стекол [18], [19], с другой стороны, на микроскопической теории ориентационного фазового перехода при $260 \mathrm{~K}$ в $\mathrm{C}_{60}$, предложенной в [20], [21].

Основная физическая проблема, которая возникает на этом пути, заключается в следующем: как описать состояние, обладающее спином или моментом (как орто-молекулы в $\mathrm{H}_{2}$ ), и состояние, не имеющее такового (как пара-молекулы), в случае одинаковых молекул $\mathrm{C}_{60}$. Оказывается возможным проследить аналогию со 
смесями, основываясь на изучении роли различных типов взаимных ориентаций молекул. В результате такого рассмотрения строится модель (в духе известной модели спинового стекла Шеррингтона-Киркпатрика [22]), в которой роль спинов играют определенные комбинации сферических гармоник. Применяя затем хорошо известные методы теории спиновых стекол к предложенной модели, мы получаем результаты, находящиеся в согласии с экспериментальными данными: сосуществование стекла и дальнего ориентационного порядка и наличие широкого максимума на кривой зависимости ориентационной части теплоемкости от температуры. Кроме того, предложенная модель позволяет рассмотреть зависимость ориентационного перехода от давления при небольших давлениях.

\section{2. ДАЛЬНИЙ ОРИЕНТАЦИОННЫЙ ПОРЯДОК}

Напомним некоторые из результатов работ [20], [21], которые понадобятся нам для построения теории ориентационного стекла. Главное наше физическое предположение заключается в том, что в соответствии со сказанным выше мы ограничиваемся рассмотрением конечного числа разрешенных ориентаций молекул вместо непрерывных вращений вокруг узлов кристаллической решетки. Эта аппроксимация является следствием того, что на кривой зависимости энергии межмолекулярного взаимодействия от углов имеются два упомянутых выше мимнимума; значения энергии этих минимумов лежат много ниже, чем остальные значения энергии. Мы принимаем во внимание только ориентации с пентагонами, гексагонами и двойными связями, направленными на 12 ближайших соседей. Таких разрешенных состояний достаточно много. Теперь матричные элементы энергии могут принимать только три значения: энергия невыгодного общего положения $J_{0}$, энергия наиболее выгодного положения $J_{\mathrm{P}}$, когда пентагон одной молекулы направлен на двойную связь другой, и энергия второго минимума $J_{\mathrm{H}}$ (гексагон против двойной связи). Следуя работе [23] и полагая $J_{0}=0$, получаем на основе расчета угловой зависимости энергии взаимодействия пары молекул [3], что $J_{\mathrm{P}}=-300 \mathrm{~K}$ и $J_{\mathrm{H}}=-110 \mathrm{~K}$. Заметим, что теоретическая кривая в [3] отражает суммарный вклад всех гармоник, и вклады по номерам $l$ не разделены. Поскольку, по существу, нас интересуют лишь трансформационные свойства функций, описывающих состояние молекулы, мы можем не использовать мультипольное разложение и в рамках нашего модельного подхода ограничиться простейшим вариантом с $l=6$.

Построим функции $P_{i}(\omega)$ и $H_{i}(\omega)$ из кубических гармоник $K_{m}=K_{6, m}, m=$ $1,2, \ldots, 13$ (см., например, [24]). Все функции $P_{i}$ и $H_{i}$ представляют собой суммы гармоник и отвечают икосаэдрической симметрии молекулы (т.е. преобразуются по единичному представлению $A_{1 g}$ группы икосаэдра $I_{h}$ ) в одной из восьми соответствующим образом повернутых систем координат. В состояниях $P_{i}\left(H_{i}\right) 6$ пентагонов (гексагонов) и 6 двойных связей направлены к 12 ближайшим соседям вдоль различных осей [100]. Функция $P_{1}(\omega)$ описывает молекулу, повернутую из стандартного $B$-положения (см. работу [8]) вокруг оси [111] на угол $97.761^{\circ}$. Соответствующий угол поворота для $H_{1}(\omega)$ равен $37.761^{\circ}$. Функции $P_{2}, P_{3}$ и $P_{4}$ (или $H_{2}$, $\left.H_{3}, H_{4}\right)$ получаются из $P_{1}\left(H_{1}\right)$ последовательными поворотами икосаэдра на $90^{\circ}$ против часовой стрелки вокруг оси $z$. 
В единой стандартной системе координат с осью $z$, направленной вдоль одной из сторон куба в ГКЦ-решетке, эти функции имеют вид

$$
\begin{gathered}
P_{1}(\omega)=\alpha_{\mathrm{P}} K_{1}(\omega)+\beta_{\mathrm{P}}\left[K_{8}(\omega)+K_{9}(\omega)+K_{10}(\omega)\right]+ \\
+\gamma_{\mathrm{P}}\left[K_{11}(\omega)+K_{12}(\omega)+K_{13}(\omega)\right] \\
P_{2}(\omega)=\alpha_{\mathrm{P}} K_{1}(\omega)+\beta_{\mathrm{P}}\left[-K_{8}(\omega)+K_{9}(\omega)-K_{10}(\omega)\right]+ \\
+\gamma_{\mathrm{P}}\left[-K_{11}(\omega)+K_{12}(\omega)-K_{13}(\omega)\right] \\
P_{3}(\omega)=\alpha_{\mathrm{P}} K_{1}(\omega)+\beta_{\mathrm{P}}\left[K_{8}(\omega)-K_{9}(\omega)-K_{10}(\omega)\right]+ \\
+\gamma_{\mathrm{P}}\left[K_{11}(\omega)-K_{12}(\omega)-K_{13}(\omega)\right] \\
P_{4}(\omega)=\alpha_{\mathrm{P}} K_{1}(\omega)+\beta_{\mathrm{P}}\left[-K_{8}(\omega)-K_{9}(\omega)+K_{10}(\omega)\right]+ \\
+\gamma_{\mathrm{P}}\left[-K_{11}(\omega)-K_{12}(\omega)+K_{13}(\omega)\right]
\end{gathered}
$$

где $\alpha_{\mathrm{P}}=-0.389, \beta_{\mathrm{P}}=0.315, \gamma_{\mathrm{P}}=-0.429$. Функции $H_{i}(\omega)$ имеют тот же вид, что и $P_{i}(\omega)$, но с коэффициентами $\alpha_{\mathrm{H}}=0.466, \beta_{\mathrm{H}}=0.377, \gamma_{\mathrm{H}}=0.344$. Все функции нормированы на единицу.

Минимизируя ориентационную часть свободной энергии, получим в приближении среднего поля нелинейное интегральное уравнение для одночастичной ориентационной функции распределения $f_{i}(\omega)$ для молекулы $\mathrm{C}_{60}$, расположенной в $i$-й подрешетке ГКЦ-решетки. Тогда вблизи точки ветвления $T_{\mathrm{b}}$ (подробнее см. в [20]) можно записать линеаризованную систему уравнений вида

$$
h_{1}(\omega)+\frac{1}{4 \pi T} \int d \omega^{\prime}\left[B\left(\omega, \omega^{\prime}\right) h_{2}\left(\omega^{\prime}\right)+A\left(\omega, \omega^{\prime}\right) h_{3}\left(\omega^{\prime}\right)+D\left(\omega, \omega^{\prime}\right) h_{4}\left(\omega^{\prime}\right)\right]=0
$$

и аналогично для трех остальных подрешеток, где $g_{i}(\omega)=\mu h_{i}(\omega)+\cdots, \mu=1 / T-$ $1 / T_{\mathrm{b}}, g_{i}(\omega)=\ln \left[f_{i}(\omega) / G_{i}\right]$, константы $G_{i}$ определяются из условий нормировки. В системе $(5) A\left(\omega, \omega^{\prime}\right), B\left(\omega, \omega^{\prime}\right), D\left(\omega, \omega^{\prime}\right)$ задают суммарные взаимодействия центральной молекулы с ближайшими соседями, относящимися к одной подрешетке (соответственно в горизонтальной и двух вертикальных плоскостях). Например, для суммы по соседям в плоскости, перпендикулярной оси $x$, имеем

$$
\begin{aligned}
D\left(\omega, \omega^{\prime}\right)=2\{ & {\left[\left(P_{1}(\omega)+P_{4}(\omega)\right) J_{\mathrm{P}}+\left(H_{1}(\omega)+H_{4}(\omega)\right) J_{\mathrm{H}}\right] \times } \\
& \times\left[P_{2}\left(\omega^{\prime}\right)+P_{3}\left(\omega^{\prime}\right)+H_{2}\left(\omega^{\prime}\right)+H_{3}\left(\omega^{\prime}\right)\right]+ \\
& +\left[P_{2}(\omega)+P_{3}(\omega)+H_{2}(\omega)+H_{3}(\omega)\right] \times \\
& \times\left[\left(P_{1}\left(\omega^{\prime}\right)+P_{4}\left(\omega^{\prime}\right)\right) J_{\mathrm{P}}+\left(H_{1}\left(\omega^{\prime}\right)+H_{4}\left(\omega^{\prime}\right)\right) J_{\mathrm{H}}\right]+ \\
& +\left[\left(P_{2}(\omega)+P_{3}(\omega)\right) J_{\mathrm{P}}+\left(H_{2}(\omega)+H_{3}(\omega)\right) J_{\mathrm{H}}\right] \times \\
& \times\left[P_{1}\left(\omega^{\prime}\right)+P_{4}\left(\omega^{\prime}\right)+H_{1}\left(\omega^{\prime}\right)+H_{4}\left(\omega^{\prime}\right)\right]+ \\
& +\left[P_{1}(\omega)+P_{4}(\omega)+H_{1}(\omega)+H_{4}(\omega)\right] \times \\
& \left.\times\left[\left(P_{2}\left(\omega^{\prime}\right)+P_{3}\left(\omega^{\prime}\right)\right) J_{\mathrm{P}}+\left(H_{2}\left(\omega^{\prime}\right)+H_{3}\left(\omega^{\prime}\right)\right) J_{\mathrm{H}}\right]\right\} .
\end{aligned}
$$

Кроме того, должно быть наложено дополнительное условие перехода функций $h_{i}(\omega)$ друг в друга при поворотах, оставляющих неизменной исходную ГКЦ-решетку. В точке ветвления $T_{\mathrm{b}}>0$ появляется нетривиальное решение нашей системы уравнений, правильно описывающее симметрию ориентационно упорядоченного состояния. Мы получаем $T_{\mathrm{b}}=275 \mathrm{~K}$ (напомним, что $\left.T_{\mathrm{c}}^{(\exp )} \approx 260 \mathrm{~K}\right)$ и

$$
h_{1}(\omega)=a P_{1}(\omega)+b H_{1}(\omega)+c K_{1}(\omega),
$$




$$
\begin{aligned}
& h_{2}(\omega)=a P_{3}(\omega)+b H_{3}(\omega)+c K_{1}(\omega), \\
& h_{3}(\omega)=a P_{4}(\omega)+b H_{4}(\omega)+c K_{1}(\omega), \\
& h_{4}(\omega)=a P_{2}(\omega)+b H_{2}(\omega)+c K_{1}(\omega),
\end{aligned}
$$

причем

$$
a \alpha_{\mathrm{P}}+b \alpha_{\mathrm{H}}+c=0 .
$$

Это решение имеет точку поворота $T_{\mathrm{t}}$, которая лежит несколько выше, чем $T_{\mathrm{b}}$. Переход первого рода со скачком параметра упорядочения имеет место при температуре, определяющейся из условия энергетической выгодности и лежащей между этими двумя точками.

Мы установили [20], [21], что имеет место переход первого рода, нашли температуру ветвления, симметрию упорядоченной фазы, а также долю $\rho_{\mathrm{P}}\left(\rho_{\mathrm{H}}\right)$ молекул, у которых пентагоны (гексагоны) обращены к двойным связям ближайших соседей вблизи фазового перехода, получив при этом хорошее согласие с экспериментальными данными

$$
\begin{aligned}
& \rho_{\mathrm{P}}=\frac{a}{a+b}=0.608, \\
& \rho_{\mathrm{H}}=\frac{b}{a+b}=0.392 .
\end{aligned}
$$

Как показывают эксперименты (см., например, [1], [2]), отношение количества молекул, находящихся в этих двух состояниях, составляет 60:40 около температуры перехода, увеличивается с понижением температуры и замерзает при $T=T_{\mathrm{g}}$. Заметим, что решение (7)-(11) включает в себя и равновероятные молекулярные ориентации $K_{1}(\omega)$, описывающие вращения в кубической решетке (см., например, [25]), в дополнение к Н- и Р-состояниям.

Уравнения, приведенные в работах [20], [21], могут быть переписаны в более компактной форме, которая нам понадобится дальше. Для этого мы используем тот факт, что в упорядоченной фазе все решетки физически эквивалентны, они переходят одна в другую при поворотах кристалла на $90^{\circ}$ вокруг оси $z$. Следовательно, выражения для $h_{2}, h_{3}, h_{4}$ в их "собственной" системе координат формально совпадают с $h_{1}$. Теперь мы можем переписать систему уравнений вида (5) в виде одного уравнения следующим образом:

$$
h_{1}(\widetilde{\omega})+\frac{1}{4 \pi T} \int d \widetilde{\omega}^{\prime} E\left(\widetilde{\omega}, \widetilde{\omega}^{\prime}\right) h_{1}\left(\widetilde{\omega}^{\prime}\right)=0,
$$

где

$$
\int d \omega^{\prime}\left[B\left(\omega, \omega^{\prime}\right) h_{2}\left(\omega^{\prime}\right)+A\left(\omega, \omega^{\prime}\right) h_{3}\left(\omega^{\prime}\right)+D\left(\omega, \omega^{\prime}\right) h_{4}\left(\omega^{\prime}\right)\right] \equiv \int d \widetilde{\omega}^{\prime} E\left(\widetilde{\omega}, \widetilde{\omega}^{\prime}\right) h_{1}\left(\widetilde{\omega}^{\prime}\right),
$$

обозначение $h_{i}(\widetilde{\omega})$ соответствует записи $h_{i}(\omega)$ в $i$-й системе координат.

Матричные элементы $E$ симметричны. Они зависят от $J_{\mathrm{P}}, J_{\mathrm{H}}$ и коэффициентов $\alpha, \beta, \gamma$. Матрицу $E$ можно диагонализовать:

$$
\begin{aligned}
& E\left(\widetilde{\omega}, \widetilde{\omega}^{\prime}\right)=\frac{1}{4 \pi T_{\mathrm{b}}}\left\{E_{r}\left[K_{8}(\widetilde{\omega})+K_{9}(\widetilde{\omega})+K_{10}(\widetilde{\omega})\right]+E_{s}\left[K_{11}(\widetilde{\omega})+K_{12}(\widetilde{\omega})+K_{13}(\widetilde{\omega})\right]\right\} \times \\
& \quad \times\left\{E_{r}\left[K_{8}\left(\widetilde{\omega}^{\prime}\right)+K_{9}\left(\widetilde{\omega}^{\prime}\right)+K_{10}\left(\widetilde{\omega}^{\prime}\right)\right]+E_{s}\left[K_{11}\left(\widetilde{\omega}^{\prime}\right)+K_{12}\left(\widetilde{\omega}^{\prime}\right)+K_{13}\left(\widetilde{\omega}^{\prime}\right)\right]\right\} .
\end{aligned}
$$


Решение уравнения (14) имеет вид

$$
h(\widetilde{\omega})=r\left[K_{8}(\widetilde{\omega})+K_{9}(\widetilde{\omega})+K_{10}(\widetilde{\omega})\right]+s\left[K_{11}(\widetilde{\omega})+K_{12}(\widetilde{\omega})+K_{13}(\widetilde{\omega})\right] .
$$

Коэффициенты уравнений (16), (17) определяются в точке ветвления $T_{\mathrm{b}}$.

Уравнения (7)-(11) и выражение (17) дают аналитическое решение, описывающее функции распределения вблизи точки бифуркации. Нетрудно найти решение исходных нелинейных интегральных уравнений в более широкой области температур. Это решение имеет ту же форму, но коэффициенты зависят от температуры. Однако $\rho_{\mathrm{P}}$ и $\rho_{\mathrm{H}}$, получаемые таким образом при фиксированных $J_{\mathrm{P}}$ и $J_{\mathrm{H}}$, слабо зависят от температуры, что не соответствует экспериментальным данным. Дело в том, что два минимума потенциальной энергии (соответствующие Р- и Н-ориентациям) разделены высоким потенциальным барьером, и это не учитывается напрямую в нашей модели. Достаточно очевидно, что парные взаимодействия между молекулами становятся при понижении температуры более чувствительными к их взаимной ориентации. Действительно, теоретические оценки [3] показывают, что либрационная амплитуда для поворотов вокруг оси [111] увеличивается почти в два раза, когда температура изменяется от $T_{\mathrm{g}}$ до $T_{\mathrm{c}}$. Попробуем ввести зависимость $J_{\mathrm{P}}=J_{\mathrm{P}}(T)$ и $J_{\mathrm{H}}=J_{\mathrm{H}}(T)$, чтобы подправить нашу модель [23]. Действительно, можно описать экспериментальные данные из работ [1], [2], используя наше решение (7)-(11) и формулу (17) следующим образом. Изменяя $J_{\mathrm{P}}$ и $J_{\mathrm{H}}$ и решая наши уравнения для разных значений, мы можем найти величины $J_{\mathrm{P}}(T)$ и $J_{\mathrm{H}}(T)$, которые дают правильные экспериментальные значения $\rho_{\mathrm{P}}(T)$ и $\rho_{\mathrm{H}}(T)$, т.е. решить своего рода обратную задачу.

В связи с полученными значениями получении величин $J_{\mathrm{P}}(T)$ и $J_{\mathrm{H}}(T)$ важно отметить следующее. Как следует из эксперимента (см., работы [1], [13]), существует небольшая аномалия при $T \approx 150 \mathrm{~K}$, причем в настоящее время не ясно, соответствует ли эта температура прекращению некоторых вращений или какому-либо другому явлению. Полученная нами “подгонкой” под эксперимент нелинейная температурная зависимость параметров $J_{\mathrm{H}}$ и $J_{\mathrm{P}}$ такова, что, когда $T_{\mathrm{b}} \rightarrow 150 \mathrm{~K}$ сверху $\left(\right.$ а $\rho_{\mathrm{P}} \rightarrow 0.7$ снизу), величина $J_{\mathrm{H}}$ стремится к нулю, а $J_{\mathrm{P}}$ остается отрицательной. Выше этой температуры оба типа взаимных ориентаций молекул являются выгодными. Выше концентрации $\rho_{\mathrm{P}}=0.7$ появляется фрустрация: Р-состояния остаются выгодными, но Н-состояния оказываются невыгодными. Это является следствием косвенного учета потенциального барьера между Р- и Н-состояниями с помощью введения зависимости эффективных параметров $J_{\mathrm{P}}$ и $J_{\mathrm{H}}$ от температуры.

\section{3. ОРИЕНТАЦИОННОЕ СТЕКЛО}

Используя полученные качественные полуфеноменологические результаты, можно описать физическую наблюдаемую картину следующим образом. При высоких температурах молекулы вращаются почти свободно вокруг своих центров. При понижении температуры возможность для молекулы изменить ориентацию уменьшается из-за наличия высокого потенциального барьера между наинизшими ориентационными состояниями пентагон-двойная связь и гексагон-двойная связь. Поэтому молекул в менее выгодном Н-состоянии много меньше $(\approx 17 \%)$, чем в Р-состоянии. 
Принято считать, что стекло возникает тогда, когда температура уже не достаточна для преодоления потенциального барьера [1], [2], [9]-[12].

Мы хотим обратить внимание на существование фрустрации, связанной с тем, что взаимодействие молекулы с соседней, если на эту соседнюю молекулу "смотрит" гексагон, является невыгодным, а если пентагон, - выгодным. Каждая молекула, находящаяся в Н-состоянии, имеет 6 гексагонов, взаимодействие которых с соседями невыгодно по сравнению с пентагонами, и 6 двойных связей. Последние могут приводить к энергетически выгодным взаимодействиям, поскольку могут быть обращены к пентагонам соседних молекул. Р-состояния являются выгодными как со стороны пентагонов, так и со стороны двойных связей. Поэтому поведение системы при низких температурах можно считать подобным поведению разбавленных молекулярных кристаллов.

Теперь, когда мы показали, что в рассматриваемой системе при низких температурах присутствуют фрустрация и беспорядок, считающиеся главными характерными чертами спиновых стекол, мы можем построить модель ориентационного стекла в $\mathrm{C}_{60}$. Это может быть проделано в полной аналогии с тем, как в пионерских работах по теории спиновых стекол [22], [26] обычная модель Изинга преобразуется в модель Шеррингтона-Киркпатрика. Подобно тому как в работах [18], [19] этот подход был обобщен путем замены изинговского спина на квадрупольный момент, теперь мы заменяем спин линейной комбинацией гармоник (17). Затем мы применяем к полученной модели стандартные методы теории спиновых стекол (см., например, [27]), в том числе метод реплик и представление о нарушенной репличной симметрии. Так как некоторые из интегралов вида

$$
\langle k, m, n\rangle=\int d \omega K_{k}(\omega) K_{m}(\omega) K_{n}(\omega)
$$

отличны от нуля, мы имеем $\psi^{3}$-ную модель и в результате получаем сосуществование ориентационного стекла и дальнего ориентационного порядка, как показано в работах [18], [19], [28]. Наше дальнейшее рассмотрение наиболее близко следует работе [28].

Итак, рассмотрим систему частиц, находящихся в узлах $i, j$ решетки, их случайное взаимодействие зададим с помощью гамильтониана

$$
H=-\frac{1}{2} \sum_{i \neq j} J_{i j} \widehat{U}_{i} \widehat{U}_{j},
$$

где $J_{i j}$ распределены с вероятностью

$$
P\left(J_{i j}\right)=\frac{1}{\sqrt{2 \pi J}} \exp \left[-\frac{J_{i j}^{2}}{2 J^{2}}\right] .
$$

Мы будем считать, что $J=\tilde{J} / \sqrt{N}$, так что справедливо приближение среднего поля, а

$$
\widehat{U}=c\left[K_{8}^{i}(\widetilde{\omega})+K_{9}^{i}(\widetilde{\omega})+K_{10}^{i}(\widetilde{\omega})\right]+d\left[K_{11}^{i}(\widetilde{\omega})+K_{12}^{i}(\widetilde{\omega})+K_{13}^{i}(\widetilde{\omega})\right]
$$


Вообще говоря, коэффициенты $c$ и $d$ зависят от отношения числа Р- и Н-состояний, которое в свою очередь зависит от температуры, и для описания всей фазовой диаграммы эта зависимость должна быть принята во внимание. Однако в низкотемпературной фазе стекла они постоянны, и мы будем считать, что $d / c=-0.914$, $\rho_{\mathrm{P}}=0.83$, как при $T=T_{\mathrm{g}}$.

Используя метод реплик, можно записать свободную энергию, усредненную по беспорядку, в виде

$$
\begin{aligned}
\frac{\langle F\rangle_{J}}{N k T}=\lim _{n \rightarrow 0} \frac{1}{n} \max \left\{\frac{t^{2}}{4} \sum_{\alpha}\left(p^{\alpha}\right)^{2}+\frac{t^{2}}{2} \sum_{\alpha>\beta}\left(q^{\alpha \beta}\right)^{2}-\right. \\
\left.\quad-\ln \operatorname{Tr} \exp \left[\frac{t^{2}}{2} \sum_{\alpha} p^{\alpha}\left(\widehat{U}^{\alpha}\right)^{2}+t^{2} \sum_{\alpha>\beta} q^{\alpha \beta} \widehat{U}^{\alpha} \widehat{U}^{\beta}\right]\right\} .
\end{aligned}
$$

Здесь $t=\tilde{J} / k T, \operatorname{Tr}(\ldots) \equiv \int_{0}^{2 \pi} d \varphi \int_{0}^{\pi} d \cos (\theta)(\ldots)$, числа $\alpha$ и $\beta$ нумеруют реплики.

Стандартные условия для седловой точки свободной энергии определяют параметр порядка стекла $q$ и регулярный параметр порядка $x$ :

$$
\begin{aligned}
q^{\alpha \beta} & =\frac{\operatorname{Tr}\left(\widehat{U}^{\alpha} \widehat{U}^{\beta} e^{\hat{\theta}}\right)}{\operatorname{Tr} e^{\hat{\theta}}}, \\
x^{\alpha} & =\frac{\operatorname{Tr}\left(\widehat{U}^{\alpha} e^{\hat{\theta}}\right)}{\operatorname{Tr} e^{\hat{\theta}}},
\end{aligned}
$$

а также вспомогательный параметр порядка

$$
p^{\alpha}=\frac{\operatorname{Tr}\left(\left(\widehat{U}^{\alpha}\right)^{2} e^{\hat{\theta}}\right)}{\operatorname{Tr} e^{\hat{\theta}}} .
$$

Здесь

$$
\hat{\theta}=\frac{t^{2}}{2} \sum_{\alpha} p^{\alpha}\left(\widehat{U}^{\alpha}\right)^{2}+t^{2} \sum_{\alpha>\beta} q^{\alpha \beta} \widehat{U}^{\alpha} \widehat{U}^{\beta} .
$$

В приближении симметричных реплик (RS) свободная энергия (22) принимает вид

$$
F_{\mathrm{RS}}=-N k T\left\{t^{2} \frac{q^{2}}{4}-t^{2} \frac{p^{2}}{4}+\int_{-\infty}^{\infty} \frac{d z}{\sqrt{2 \pi}} e^{-z^{2} / 2} \ln \operatorname{Tr} e^{\hat{\theta}_{\mathrm{RS}}}\right\} .
$$

Здесь

$$
\hat{\theta}_{\mathrm{RS}}=z t \sqrt{q} \widehat{U}+t^{2} \frac{p-q}{2} \widehat{U}^{2} .
$$

Из условий экстремума для свободной энергии (27) получаем уравнения для параметров порядка:

$$
\begin{aligned}
& q_{\mathrm{RS}}=\int d z^{\mathrm{G}}\left\{\frac{\operatorname{Tr}\left(\widehat{U} e^{\hat{\theta}_{\mathrm{RS}}}\right)}{\operatorname{Tr} e^{\hat{\theta}_{\mathrm{RS}}}}\right\}^{2}, \\
& x_{\mathrm{RS}}=\int d z^{\mathrm{G}} \frac{\operatorname{Tr}\left(\widehat{U} e^{\hat{\theta}_{\mathrm{RS}}}\right)}{\operatorname{Tr} e^{\hat{\theta}_{\mathrm{RS}}}}, \\
& p_{\mathrm{RS}}=\int d z^{\mathrm{G}} \frac{\operatorname{Tr}\left(\widehat{U}^{2} e^{\hat{\theta}_{\mathrm{RS}}}\right)}{\operatorname{Tr} e^{\hat{\theta}_{\mathrm{RS}}}} .
\end{aligned}
$$




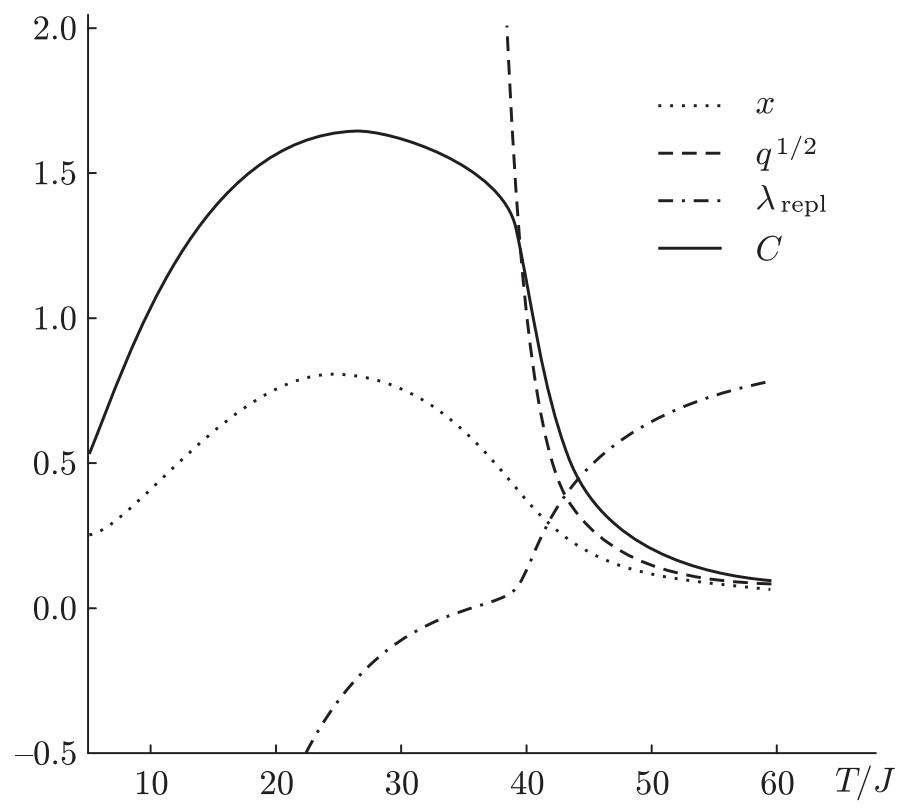

Рис. 1. Зависимость RS-параметров порядка и ориентационного вклада в теплоемкость от температуры. Здесь $d / c=-0.914$, сплошная кривая описывает теплоемкость $C$, пунктирная и штриховая кривые отвечают параметрам порядка $x$ и $\sqrt{q}$ соответственно, штрихпунктирная кривая описывает $\lambda_{\text {repl }}$. Симметрия реплик нарушается при температуре $T_{0}$, соответствующей $\lambda_{\text {repl }}=0$.

Здесь

$$
\int d z^{\mathrm{G}}=\int_{-\infty}^{\infty} \frac{d z}{\sqrt{2 \pi}} \exp \left(-\frac{z^{2}}{2}\right) .
$$

Выражение для ориентационного вклада в теплоемкость имеет вид

$$
\frac{C_{v(\mathrm{RS})}}{k N}=\frac{d}{d(k T / \tilde{J})}\left[\frac{\tilde{J}}{k T} \frac{q^{2}-p^{2}}{2}\right] .
$$

Репликосимметричное решение стабильно, пока репликонная мода $\lambda$ отлична от нуля [27]. Для нашей модели

$$
\lambda_{\text {repl }}=1-t^{2} \int d z^{\mathrm{G}}\left\{\frac{\operatorname{Tr}\left(\widehat{U}^{2} e^{\hat{\theta}_{\mathrm{RS}}}\right)}{\operatorname{Tr} e^{\hat{\theta}_{\mathrm{RS}}}}-\left[\frac{\operatorname{Tr}\left(\widehat{U} e^{\hat{\theta}_{\mathrm{RS}}}\right)}{\operatorname{Tr} e^{\hat{\theta}_{\mathrm{RS}}}}\right]^{2}\right\}^{2} .
$$

Результаты расчетов представлены на рисунке. Параметры порядка отличны от нуля при всех температурах, поскольку $\int d \omega u^{2 k+1}(\omega) \neq 0, k=1,2, \ldots$ [28]. Одновременное сосуществование ориентационного упорядочения (параметр порядка $x \neq 0$ ) и стекла (параметр порядка $q \neq x^{2}$ ) соответствует экспериментальным данным из работ [1], [2]. Для ориентационного вклада в теплоемкость мы имеем плавную кривую с широким максимумом, что качественно соответствует данным для $C_{p}(T)$, полученным экспериментально [9], [15], [16], [29]-[31]. 
Нарушение репличной симметрии происходит при температуре $T_{0}$, когда RS-peшение становится неустойчивым. Эта температура определяется условием $\lambda_{\text {repl }}=0$. В моделях подобного типа именно эту температуру считают температурой перехода в стекло. Она несколько выше температуры максимума на кривой теплоемкости, и это также соответствует эксперименту.

Пользуясь стандартной процедурой (см., например, [27]), проведем первый этап нарушения репличной симметрии по Паризи (1RSB) $(n$ реплик разбиваются на $n / m$ групп по $m$ реплик в каждой) и получим следующее выражение для свободной энергии:

$$
\begin{aligned}
F_{1 \mathrm{RSB}}=- & N k T\left\{m t^{2} \frac{r_{1}^{2}}{4}+(1-m) t^{2} \frac{\left(r_{1}+v\right)^{2}}{4}-\right. \\
& \left.-t^{2} \frac{p^{2}}{4}+\frac{1}{m} \int d z^{\mathrm{G}} \int d s^{G}\left(\operatorname{Tr} e^{\hat{\theta}_{1 \mathrm{RSB}}}\right)^{m}\right\} .
\end{aligned}
$$

Здесь

$$
\hat{\theta}_{1 \mathrm{RSB}}=z t \sqrt{r_{1}} \widehat{U}+s t \sqrt{v} \widehat{U}+t^{2} \frac{p-r_{1}-v}{2} \widehat{U}^{2} .
$$

Условия экстремума для $F_{1 \mathrm{RSB}}$ дают уравнения для параметров порядка стекла $r_{1}$ и $v$, регулярного параметра порядка $x$, вспомогательного параметра порядка $p$ и параметра $m$.

Соответствующие выражения для энтропии и теплоемкости имеют вид

$$
\begin{aligned}
S_{1 \mathrm{RSB}}=N k & \left\{m t^{2} \frac{3 r_{1}^{2}}{4}+(1-m) t^{2} \frac{3\left(r_{1}+v\right)^{2}}{4}-t^{2} \frac{3 p^{2}}{4}+\right. \\
& \left.+\frac{1}{m} \int d z^{\mathrm{G}} \int d s^{G}\left(\operatorname{Tr} e^{\hat{\theta}_{1 \mathrm{RSB}}}\right)^{m}\right\}, \\
\frac{C_{v(1 \mathrm{RSB})}}{k N}= & \frac{d}{d(k T / \tilde{J})}\left[\frac{\tilde{J}}{k T} \frac{m^{2} r_{1}^{2}+(1-m)\left(r_{1}+v\right)^{2}-p^{2}}{2}\right] .
\end{aligned}
$$

Чтобы оценить вид 1RSB-решения вблизи той точки, где оно перестает совпадать c RS-решением, т.е. в окрестности $T_{0}$, разложим выражение для свободной энергии до третьего порядка включительно, считая отклонения $\delta q^{\alpha \beta}$ от $q_{\mathrm{RS}}$ малыми. Нам будет удобно исходить из выражения (22). Отклонение $\Delta F$ свободной энергии задается формулой

$$
\begin{aligned}
& \frac{\Delta F}{N k T}=\lim _{n \rightarrow 0} \frac{1}{n}\left\{\frac{t^{2}}{2} \sum_{\alpha>\beta}\left(\delta q^{\alpha \beta}\right)^{2}-\frac{1}{2} \frac{\operatorname{Tr}\left(\widehat{A}^{2} e^{\widehat{\Xi}}\right)}{\operatorname{Tr} e^{\widehat{\Xi}}}+\frac{1}{2}\left[\frac{\operatorname{Tr}\left(\widehat{A} e^{\widehat{\Xi}}\right)}{\operatorname{Tr} e^{\widehat{\Xi}}}\right]^{2}-\frac{1}{6} \frac{\operatorname{Tr}\left(\widehat{A}^{3} e^{\widehat{\Xi}}\right)}{\operatorname{Tr} e^{\widehat{\Xi}}}+\right. \\
& \left.+\frac{1}{2} \frac{\operatorname{Tr}\left(\widehat{A} e^{\widehat{\Xi}}\right)}{\operatorname{Tr} e^{\widehat{\Xi}}} \frac{\operatorname{Tr}\left(\widehat{A}^{2} e^{\widehat{\Xi}}\right)}{\operatorname{Tr} e^{\widehat{\Xi}}}-\frac{1}{3}\left[\frac{\operatorname{Tr}\left(\widehat{A} e^{\widehat{\Xi}}\right)}{\operatorname{Tr} e^{\hat{\Xi}}}\right]^{3}+\cdots\right\} .
\end{aligned}
$$

Здесь

$$
\widehat{A}=t^{2} \sum_{\alpha>\beta} \delta q^{\alpha \beta} \widehat{U}^{\alpha} \widehat{U}^{\beta}, \quad \widehat{\Xi}=\frac{t^{2}}{2} p \sum_{\alpha}\left(\widehat{U}^{\alpha}\right)^{2}+t^{2} q_{\mathrm{RS}} \sum_{\alpha>\beta} \widehat{U}^{\alpha} \widehat{U}^{\beta} .
$$

Используя формулы приложения 1 , получаем

$$
\frac{\Delta F}{N k T}=\frac{t^{2}}{4}\left(1-t^{2} W\right)\left\{-[r-(m-1) v]^{2}+v^{2} m(m-1)\right\}+\frac{t^{4}}{2} Q[r-(m-1) v]^{2}-
$$




$$
\begin{aligned}
& -t^{6}\left\{C[r-(m-1) v]^{3}+D[r-(m-1) v] v^{2} m(m-1)-\right. \\
& \left.-B_{3} v^{3} m^{2}(m-1)+B_{4} v^{3} m(m-1)(2 m-1)\right\}+\cdots .
\end{aligned}
$$

Здесь $t=t_{0}+\Delta t, r_{1}=q_{\mathrm{RS}}+r$, а выражения для параметров $W, Q, C, D, B_{3}, B_{4}$ даны в приложении 2 .

Из условий экстремума для свободной энергии (41) и в силу того, что $\left.Q\right|_{t=t_{0}} \neq 0$, получаем условие ветвления $r-(m-1) v=0+o(\Delta t)^{2}$, т.е. условие отсутствия линейного члена для параметров порядка стекла. Другого члена, линейного по параметру порядка стекла, нет, поскольку $\left.\left(1-t^{2} W\right)\right|_{t=t_{0}}=\left.\lambda_{\text {repl }}\right|_{t=t_{0}}=0$ в точке ветвления. Окончательно получаем

$$
\begin{gathered}
2\left[-\frac{t_{0}}{2}-\left.\frac{t_{0}^{4}}{4} \frac{d W}{d t}\right|_{t=t_{0}}\right] \Delta t=t_{0}^{6}\left[-B_{4}+m\left(-B_{3}+2 B_{4}\right)\right] v \\
(2 m-1)\left[-\frac{t_{0}}{2}-\left.\frac{t_{0}^{4}}{4} \frac{d W}{d t}\right|_{t=t_{0}}\right] \Delta t=t_{0}^{6}\left\{(2 m-1)\left[-B_{4}+m\left(-B_{3}+2 B_{4}\right)\right]+\right. \\
\left.+m(m-1)\left(-B_{3}+2 B_{4}\right)\right\} v
\end{gathered}
$$

Здесь $B_{3}$ и $B_{4}$ берутся при $T=T_{0}$. Отсюда получаем, что в окрестности точки $T_{0}$ появления 1RSB-решения

$$
v=\frac{2+\left.t_{0}^{3} \frac{d W}{d t}\right|_{t=t_{0}}}{12 B_{4}(1-m) t_{0}^{5}} \Delta t, \quad r=(m-1) v
$$

и в точке ветвления $T_{0}$

$$
m=\frac{B_{4}}{B_{3}} .
$$

Заметим, что все полученные нами выражения верны для гамильтониана (19), где $\widehat{U}-$ произвольный диагональный оператор такой, что $\operatorname{Tr} \widehat{U}=0$, a $\operatorname{Tr} \widehat{U}^{3}$ и $\left.Q\right|_{t=t_{0}}$ отличны от нуля.

Для нашей модели с $\widehat{U}$, определяемым выражением (21), из приведенных выше формул можно получить, что $m=0.099$, и скачка параметров упорядочения в точке появления 1RSB-решения нет $(m<1)$. Кроме того, кривая теплоемкости мало меняется при переходе от RS- к 1RSB-решению.

Заметим, что наш подход оказался успешным и для нахождения характеристик ориентационной фазовой диаграммы молекулярного $\mathrm{C}_{60}$ в зависимости от давления при не очень больших давлениях, когда зависимость $J_{\mathrm{P}}$ и $J_{\mathrm{H}}$ от давления можно считать линейной.

\section{4. ЗАКЛЮЧЕНИЕ}

В настоящей работе мы применили полученные нами ранее результаты по ориентационному упорядочению в фуллерите $\mathrm{C}_{60}$ для построения модели низкотемпературной фазы ориентационного стекла. Мы показали, что в рассматриваемой системе присутствуют беспорядок и фрустрация, и использовали основные представления теории спиновых стекол, в том числе метод реплик и понятие нарушенной репличной симметрии. В результате мы получили, что в системе состояние стекла сосуществует с дальним ориентационным порядком, а вблизи перехода температурная зависимость ориентационной части теплоемкости имеет широкий максимум, что соответствует экспериментальным данным. 
ПРИЛОЖЕНИЕ 1

Отличны от нуля только следующие суммы:

$$
\begin{aligned}
& \lim _{n \rightarrow 0} \frac{1}{n} \sum_{\alpha, \beta}^{\prime}\left(\delta q^{\alpha \beta}\right)^{3}=(m-1) \eta^{3}-m \xi^{3} ; \\
& \lim _{n \rightarrow 0} \frac{1}{n} \sum_{\alpha, \beta, \gamma}^{\prime} \delta q^{\alpha \beta} \delta q^{\beta \gamma} \delta q^{\gamma \alpha}=(m-1)(m-2) \eta^{3}-3 m(m-1) \eta \xi^{2}+2 m^{2} \xi^{3} \\
& \lim _{n \rightarrow 0} \frac{1}{n} \sum_{\alpha, \beta, \gamma}^{\prime}\left(\delta q^{\alpha \beta}\right)^{2} \delta q^{\alpha \gamma}=(m-1)^{2} \eta^{3}-m(m-1)\left(\eta \xi^{2}+\eta^{2} \xi\right)+m^{2} \xi^{3} \\
& \lim _{n \rightarrow 0} \frac{1}{n} \sum_{\alpha, \beta, \gamma, \delta}^{\prime} \delta q^{\alpha \beta} \delta q^{\alpha \gamma} \delta q^{\alpha \delta}=\lim _{n \rightarrow 0} \frac{1}{n} \sum_{\alpha, \beta, \gamma, \delta}^{\prime} \delta q^{\alpha \beta} \delta q^{\alpha \gamma} \delta q^{\beta \delta}=(m-1)^{3} \eta^{3}+ \\
& \quad+3 m^{2}(m-1) \eta \xi^{2}-3 m(m-1)^{2} \eta^{2} \xi-m^{3 \xi^{3}} .
\end{aligned}
$$

Здесь $\eta=r+v, \xi=r$. Штрих у знака суммы означает, что в $\sum^{\prime}$ обязательно различны только индексы, принадлежащие одному и тому же $\delta q$.

\section{ПРИЛОЖЕНИЕ 2}

Здесь мы приводим формулы для расчета параметров:

$$
\begin{aligned}
W= & \left\langle\widehat{U}_{1}^{2} \widehat{U}_{2}^{2}\right\rangle-2\left\langle\widehat{U}_{1}^{2} \widehat{U}_{2} \widehat{U}_{3}\right\rangle+\left\langle\widehat{U}_{1} \widehat{U}_{2} \widehat{U}_{3} \widehat{U}_{4}\right\rangle, \quad Q=-\left\langle\widehat{U}_{1}^{2} \widehat{U}_{2} \widehat{U}_{3}\right\rangle+\left\langle\widehat{U}_{1} \widehat{U}_{2} \widehat{U}_{3} \widehat{U}_{4}\right\rangle, \\
C= & -\left(B_{2}+B_{2}^{\prime}\right)+2 B_{3}+B_{3}^{\prime}-B_{4}, \quad D=-3 B_{3}-B_{3}^{\prime}+3 B_{4}, \\
B_{2}= & \frac{1}{2}\left\langle\widehat{U}_{1}^{2} \widehat{U}_{2}^{2} \widehat{U}_{3} \widehat{U}_{4}\right\rangle+\frac{1}{2}\left\langle\widehat{U}_{1} \widehat{U}_{2} \widehat{U}_{3} \widehat{U}_{4} \widehat{U}_{5} \widehat{U}_{6}\right\rangle-\left\langle\widehat{U}_{1}^{2} \widehat{U}_{2} \widehat{U}_{3} \widehat{U}_{4} \widehat{U}_{5}\right\rangle, \\
B_{2}^{\prime}= & \frac{1}{3}\left\langle\widehat{U}_{1} \widehat{U}_{2} \widehat{U}_{3} \widehat{U}_{4} \widehat{U}_{5} \widehat{U}_{6}\right\rangle-\frac{1}{2}\left\langle\widehat{U}_{1}^{2} \widehat{U}_{2} \widehat{U}_{3} \widehat{U}_{4} \widehat{U}_{5}\right\rangle+\frac{1}{6}\left\langle\widehat{U}_{1}^{3} \widehat{U}_{2} \widehat{U}_{3} \widehat{U}_{4}\right\rangle, \\
B_{3}= & \frac{1}{6}\left\langle\widehat{U}_{1}^{2} \widehat{U}_{2}^{2} \widehat{U}_{3}^{2}\right\rangle-\frac{1}{2}\left\langle\widehat{U}_{1}^{2} \widehat{U}_{2}^{2} \widehat{U}_{3} \widehat{U}_{4}\right\rangle-\frac{1}{6}\left\langle\widehat{U}_{1} \widehat{U}_{2} \widehat{U}_{3} \widehat{U}_{4} \widehat{U}_{5} \widehat{U}_{6}\right\rangle+\frac{1}{2}\left\langle\widehat{U}_{1}^{2} \widehat{U}_{2} \widehat{U}_{3} \widehat{U}_{4} \widehat{U}_{5}\right\rangle, \\
B_{3}^{\prime}=-\left\langle\widehat{U}_{1} \widehat{U}_{2} \widehat{U}_{3} \widehat{U}_{4} \widehat{U}_{5} \widehat{U}_{6}\right\rangle+\frac{5}{2}\left\langle\widehat{U}_{1}^{2} \widehat{U}_{2} \widehat{U}_{3} \widehat{U}_{4} \widehat{U}_{5}\right\rangle-\frac{1}{2}\left\langle\widehat{U}_{1}^{3} \widehat{U}_{2} \widehat{U}_{3} \widehat{U}_{4}\right\rangle- & \quad-\frac{3}{2}\left\langle\widehat{U}_{1}^{2} \widehat{U}_{2}^{2} \widehat{U}_{3} \widehat{U}_{4}\right\rangle+\frac{1}{2}\left\langle\widehat{U}_{1}^{3} \widehat{U}_{2}^{2} \widehat{U}_{3}\right\rangle, \\
B_{4}= & \frac{1}{3}\left\langle\widehat{U}_{1} \widehat{U}_{2} \widehat{U}_{3} \widehat{U}_{4} \widehat{U}_{5} \widehat{U}_{6}\right\rangle-\left\langle\widehat{U}_{1}^{2} \widehat{U}_{2} \widehat{U}_{3} \widehat{U}_{4} \widehat{U}_{5}\right\rangle+\frac{1}{3}\left\langle\widehat{U}_{1}^{3} \widehat{U}_{2} \widehat{U}_{3} \widehat{U}_{4}\right\rangle+ \\
& +\frac{3}{4}\left\langle\widehat{U}_{1}^{2} \widehat{U}_{2}^{2} \widehat{U}_{3} \widehat{U}_{4}\right\rangle-\frac{1}{2}\left\langle\widehat{U}_{1}^{3} \widehat{U}_{2}^{2} \widehat{U}_{3}\right\rangle+\frac{1}{12}\left\langle\widehat{U}_{1}^{3} \widehat{U}_{2}^{3}\right\rangle,
\end{aligned}
$$

где

$$
\left\langle\widehat{U}_{1}^{k} \widehat{U}_{2}^{n} \ldots\right\rangle=\int d z^{\mathrm{G}} \frac{\operatorname{Tr}\left(\widehat{U}_{1}^{k} \widehat{U}_{2}^{n} \ldots e^{\Xi}\right)}{\operatorname{Tr} e^{\Xi}} .
$$

Благодарности. Авторы выражают благодарность В. А. Давыдову, А. В. Рахманиной и В.Н. Рыжову за полезные обсуждения. Работа была выполнена при частичной поддержке РФФИ (гранты №№ 05-02-17621, 05-02-17280, 07-02-00998), Гранта Президента Российской Федерации (07-02-00998MK), Российского фонда поддержки отечественной науки, фонда “Династия", Программы РАН "Квантовая Макрофизика". 


\section{Список литературы}

[1] B. Sundqvist, Adv. Phys., 48:1 (1999), 1-134.

[2] R. Moret, Acta Cryst. A, 61 (2005), 62-76.

[3] T. Yildirim, A. B. Harris, Phys. Rev. B, 46:12 (1992), 7878-7896.

[4] S. L. Chaplot, L. Pintschovious, Internat. J. Modern Phys. B, 13:3 (1999), 217-251.

[5] P. Launois, S. Ravy, R. Moret, Internat. J. Modern Phys. B, 13:3 (1999), 253-281.

[6] P. A. Heiney, J. E. Fischer, A. R. McGhie et al., Phys. Rev. Lett., 66:22 (1991), 2911-2914.

[7] R. Sachidanandam, A. B. Harris, Phys. Rev. Lett., 67:11 (1991), 1467.

[8] A. B. Harris, Physica A, 205:1-3 (1994), 154-182.

[9] F. Gugenberger, R. Heid, C. Meingast et al., Phys. Rev. Lett., 69:26 (1992), 3774-3777.

[10] W. I. F. David, R. M. Ibberson, T. J. S. Dennis et al., Europhys. Lett., 18 (1992), 219-225.

[11] W. I. F. David, R. M. Ibberson, J. Phys.: Condens. Matter, 5:43 (1993), 7923-7928.

[12] W. I. F. David, R. M. Ibberson, T. Matsuo, Proc. Math. Phys. Sci., 442:1914 (1993), 129-146.

[13] Н. А. Аксенова, А. П. Исакина, А.И. Прохватилов, М. А. Стржемечный, Физ. низк. темn., 25:8-9 (1999), 964-975.

[14] L. Pintschovius, S. L. Chaplot, G. Roth, M. Haluska, H. Kuzmany, Phys. Scripta, 57 (1995), $102-106$.

[15] T. Matsuo, H. Suga, N. I. F. David, R. M. Ibberson et al., Solid State Commun., 83:9 (1992), $711-715$.

[16] Б. В. Лебедев, К.Б. ЖКогова, Т.А. Быкова и др., Изв. РАН. Сер. хим., 9 (1996), 2229-2233.

[17] R. C. Yu, N. Tea, M. B. Salamon et al., Phys. Rev. Lett., 68:13 (1992), 2050-2053.

[18] E. A. Lutchinskaia, V. N. Ryzhov, E. E. Tareyeva, J. Phys. C, 17:26 (1984), L665-L667; Е. А. Лучинская, В. Н. Рыжов, Е. Е. Тареева, ТМФ, 67:3 (1986), 463-469; E. A. Lutchinskaia, E. E. Tareyeva, Phys. Rev. B, 52:1 (1995), 366-373.

[19] T. I. Schelkacheva, Писъма в ЖЭТФ, 76:6 (2002), 434-436.

[20] Е. Е. Тареева, Т. И. Щелкачева, ТМФ, 121:3 (1999), 479-491.

[21] T. I. Schelkacheva, E. E. Tareyeva, Phys. Rev. B, 61:5 (2000), 3143-3146.

[22] D. Sherrington, S. Kirkpatrick, Phys. Rev. Lett., 35:26 (1975), 1792-1796; S. Kirkpatrick, D. Sherrington, Phys. Rev. B, 17:11 (1978), 4384-4403.

[23] S. Lapinskas, E. E. Tornau, A. Rosengren, Phys. Rev. B, 49:14 (1994), 9372-9375.

[24] R. Heid, Phys. Rev. B, 47:23 (1993), 15912-15922.

[25] В. Л. Аксенов, Ю. А. Осипьян, В. С. Шахматов, Писъма в ЖЭЭФ, 64:2 (1996), 110-115.

[26] S. F. Edwards, P. W. Anderson, J. Phys. F, 5:5 (1975), 965-974.

[27] M. Mézard, G. Parisi, M. Virasoro, Spin Glass Theory and Beyond, Lecture Notes in Phys., 9, World Scientific, Singapore, 1987.

[28] N. V. Gribova, V. N. Ryzhov, T. I. Schelkacheva, E. E. Tareyeva, Phys. Lett. A, 315:6 (2003), $467-473$.

[29] B. V. Lebedev, A. V. Markin, V. A. Davydov et al., Thermochimica Acta, 399:1-2 (2003), 99-108.

[30] V. A. Davydov, L. S. Kashevarova, A. V. Rakhmanina et al., Phys. Rev. B, 61:18 (2000), 11936-11945.

[31] A. V. Markin, N. N. Smirnova, B. V. Lebedev et al., Thermochimica Acta, 411:1 (2004), 101-108. 